\title{
Race as a Factor in Donor Selection and Survival of Children with Hematologic Malignancies Undergoing Hematopoietic Stem Cell Transplant in Florida
}

\author{
Biljana Horn ${ }^{1}$, Deepak Chellapandian ${ }^{2}$, Nikhil Lamba ${ }^{1}$, Gauri Sunkersett ${ }^{2}$, Jorge \\ GalvezSilva $^{3}$, Edward Ziga ${ }^{4}$, Warren Alperstein ${ }^{4}$, Micahel Joyce ${ }^{5}$, Paul Castillo ${ }^{1}$, John \\ Fort $^{1}$, Jing Zhao ${ }^{1}$, and Benjamin Oshrine ${ }^{2}$ \\ ${ }^{1}$ University of Florida \\ ${ }^{2}$ Johns Hopkins All Children's Hospital \\ ${ }^{3}$ Nicklaus Children's Hospital \\ ${ }^{4}$ University of Miami Miller School of Medicine \\ ${ }^{5}$ Nemours Children's Health System
}

April 9, 2021

\begin{abstract}
Background Previous studies have explored post-hematopoietic cell transplant (HCT) outcomes by race in adults; however, pediatric data addressing this topic are scarce. Procedure This retrospective registry study included 238 White (W) and 57 Black (B) children with hematologic malignancies (HM) receiving first allogeneic HCT between 2010 and 2019 in one of five Florida pediatric HCT centers. Results We found no differences between W and B children by transplant characteristics, other than donor type. There was a significant difference in use of HLA-mismatched donors (HLA-MMD) (53\% W, 71\% B, p=0.01). When comparing HLA-MMD use to fully HLA-matched donors, B had RR of 1.47 [95\% CI 0.7-3] of receiving a mismatched unrelated donor (MMUD), RR of 2.34 [95\% CI 1.2-4.4] of receiving a mismatched related donor (MMRD), and a RR of 1.9 [95\% CI 0.99-3.6] of receiving a mismatched cord blood donor (MMCBD) HCT, respectively. There was no significant difference in the incidence of aGVHD $(48 \% \mathrm{~W}, 35 \% \mathrm{~B}), \mathrm{p}=0.1$ or cGVHD $(19 \% \mathrm{~W} 28 \% \mathrm{~B}, \mathrm{p}=0.1)$, or primary cause of death. Overall 24-month survival was $61 \%$ [95\% CI 54-68\%] for W, and 60\% [95\% CI 38-68] for B children, log-rank p=0.72. While HLA matching improved survival in $\mathrm{W}$ children, the number of B children receiving HLA-matched HCT was too small to identify the impact of HLA matching on survival. Conclusions In this contemporary cohort of children with HM we found that B children were more likely to receive HLA-MMD transplants, but this did not adversely affect survival or GVHD rates.
\end{abstract}

Race as a Factor in Donor Selection and Survival of Children with Hematologic Malignancies Undergoing Hematopoietic Stem Cell Transplant in Florida

Biljana Horn, MD ${ }^{1}$, Nikhil Lamba, BS ${ }^{1}$, Deepak Chellapandian, MDMD MBBS ${ }^{2}$ Gauri Sunkersett, DO $^{2}$, Jorge Galvez Silva, $\mathrm{MD}^{3}$, Edward Ziga, $\mathrm{MD}^{4}$, Warren Alperstein, $\mathrm{MD}^{4}$, Michael Joyce, MD, $\mathrm{PhD}^{5}$, Paul Castillo, $\mathrm{MD}^{1}$, John Fort, $\mathrm{MD}^{1}$, Jing Zhao, PhD, MS. ${ }^{1}$ and Benjamin Oshrine, MD ${ }^{2}$

Affiliations:

(1) University of Florida, Gainesville, FL

(2) Center for Cell and Gene Therapy for Non Malignant-Conditions, Blood and Marrow Transplant Program, Cancer \& Blood Disorders Institute, Johns Hopkins All Children's Hospital, St Petersburg, FL; (3) Nicklaus Children's Hospital, Miami, FL 
(4) Pediatric Hematology and Oncology, University of Miami School of Medicine, Miami, FL (5) Nemours Children's Clinic and Wolfson Children's Hospital, Jacksonville, FL

Corresponding Author:

Biljana Horn, e-mail: Biljana.horn@ufl.edu

1600 SW Archer Avenue, Gainesville, FL 32610

Phone (mobile) 415-215-6930, FAX: 3522948091

Article Type: Research Article

Word Count: abstract 249

Word Count: main text 2152

Tables: 2, Figures: 2, References: 17

Short Title: Race and outcomes in children undergoing HCT

Key Words: race disparities, pediatric HCT, malignant disorders, Florida consortium

Abbreviations used in the manuscript:

\begin{tabular}{ll}
\hline ATG & Anti-thymocyte globulin \\
\hline B & Black race \\
BM & Bone Marrow \\
CIBMTR & Center for International Blood and Marrow Transplant Research \\
FPBCC & Florida Pediatric Bone Marrow Transplant and Cell Therapy Consortium \\
GVHD, aGVHD, cGVHD & Graft-versus-host disease, acute graft versus-host disease, chronic graft-versus-host disease \\
HCT & Hematopoietic cell transplant \\
HLA & Human leukocyte antigen \\
HM & Hematologic malignancies \\
MMD & Mismatched donor \\
MMUD & Mismatched unrelated donor \\
MMRD & Mismatched related donor \\
MRD & Matched related donor \\
MUD & Matched unrelated donor \\
OS & Overall survival \\
PB & Peripheral blood \\
PTCy & Post-transplant cyclophosphamide \\
RR & Relative risk \\
UCB & Umbilical cord blood \\
W & White race \\
\hline
\end{tabular}

Previous Presentations: Accepted as Oral presentation at the 2021 PTCT at ASPHO meeting. Title: "Race as a factor in outcomes of hematopoietic stem cell transplant (HCT) for Children with Hematologic Malignancies (HM) in Florida."

\section{Abstract \\ Background}

Previous studies have explored post-hematopoietic cell transplant (HCT) outcomes by race in adults; however, pediatric data addressing this topic are scarce.

\section{Procedure}


This retrospective registry study included 238 White (W) and 57 Black (B) children with hematologic malignancies (HM) receiving first allogeneic HCT between 2010 and 2019 in one of five Florida pediatric HCT centers.

\section{Results}

We found no differences between $\mathrm{W}$ and $\mathrm{B}$ children by transplant characteristics, other than donor type. There was a significant difference in use of HLA-mismatched donors (HLA-MMD) ( $53 \% \mathrm{~W}, 71 \% \mathrm{~B}, p=0.01)$. When comparing HLA-MMD use to fully HLA-matched donors, B had RR of 1.47 [95\% CI 0.7-3] of receiving a mismatched unrelated donor (MMUD), RR of 2.34 [95\% CI 1.2-4.4] of receiving a mismatched related donor (MMRD), and a RR of 1.9 [95\% CI 0.99-3.6] of receiving a mismatched cord blood donor (MMCBD) HCT, respectively. There was no significant difference in the incidence of aGVHD $(48 \% \mathrm{~W}, 35 \% \mathrm{~B}), \mathrm{p}=0.1$ or cGVHD $(19 \% \mathrm{~W} 28 \% \mathrm{~B}, p=0.1)$, or primary cause of death. Overall 24-month survival was $61 \%$ [95\% CI $54-68 \%$ ] for $\mathrm{W}$, and $60 \%$ [95\% CI 38-68] for B children, log-rank $\mathrm{p}=0.72$. While HLA matching improved survival in $\mathrm{W}$ children, the number of $\mathrm{B}$ children receiving HLA-matched HCT was too small to identify the impact of HLA matching on survival.

\section{Conclusions}

In this contemporary cohort of children with HM we found that B children were more likely to receive HLA-MMD transplants, but this did not adversely affect survival or GVHD rates.

\section{Introduction}

Racial disparities in outcomes of children with acute lymphoblastic leukemia treated from late 1970's until 2014 have been well documented with Black, Hispanic and Native American children having lower overall survival compared to White non-Hispanic patients. (1-4). Similarly, racial and ethnic differences in pediatric acute myeloid leukemia have also been observed with inferior survival observed among Black and Hispanic children compared to White non-Hispanic children.(5).Bhatia postulated that causes of these disparities in outcomes in children with cancer are multifactorial and include advanced stage at diagnosis, disease biology, poor treatment response, non adherence to therapy, health behaviors, lower socio-economic status, differences in enrollment in upfront clinical trials and differences in health insurance (6). Analysis of a large Center for International Blood and Marrow Transplant Registry (CIBMTR) cohort of pediatric and adult patients with HM, transplanted between 1995 and 2004 who received HCT from unrelated donors, indicated worse overall survival of African-American HCT recipients, compared to Whites and the difference was not fully explained by transplant-related factors or socio-economic status emphasizing again a complex, multifactorial etiology of those differences.(7)

Given the paucity of data on the impact of race on outcomes after HCT in children, we analyzed and present data from a contemporary cohort of children receiving first allogeneic HCT for hematologic malignancies in 5 Florida pediatric HCT centers between 2010 and 2019.

\section{Method}

Data for this report come from the Florida Pediatric Bone Marrow Transplant and Cell Therapy Consortium (FPBCC). The objectives of FPBCC are to identify best pediatric HCT practices and improve survival of children receiving HCT in Florida. FPBCC was founded in 2018 and comprises 5 of the 6 pediatric transplant programs in the state of Florida, USA. All FPBCC participating centers signed memoranda of understanding and data use agreements and obtained institution-specific IRB approvals for this retrospective data analysis. Data from participating centers were downloaded from the enhanced data back to center, a CIBMTR platform, which has access to a limited CIBMTR dataset. Data were forwarded to the FPBCC statistical center, housed at the University of Florida (Gainesville, FL, USA), where data were combined into a single data set and analyzed. All participants signed an informed consent for CIBMTR data collection. Transplant centers report detailed data on consecutive hematopoietic cell transplantations to the statistical center of CIBMTR, and compliance with this reporting is monitored by on-site audits. 
We describe demographics of pediatric HCT recipients, including gender, age, race, as reported to the CIBMTR. Ethnic origin (e.g. Hispanic) was not available in our dataset. Pre-transplant characteristics included diagnosis, status of disease at transplant, time from diagnosis to transplant, performance score, and number of pre-transplant comorbidities. Transplant characteristics consist of type of donor, stem cell source, Human Leukocyte Antigen (HLA) match, conditioning regimen, and regimen intensity. Outcomes data include length of survival, cause of death, and incidence and grade of GVHD. Myeloablative regimens were defined as those using one or more of the following: total body irradiation $>500$ cGy for a single dose or $>800$ cGy for fractionate, busulfan $>7.2 \mathrm{mg} / \mathrm{kg}$ iv, melphalan $>150 \mathrm{mg} / \mathrm{m}^{2}$, and thiotepa [?]10

$\mathrm{mg} / \mathrm{kg}$. Reduced intensity regimens were defined as those using melphalan [?]150 mg/m², thiotepa $<10$ $\mathrm{mg} / \mathrm{kg}$, TBI $>200 \mathrm{cGy}$ and [?]500 cGy as a single dose or [?]800 cGy fractionated, busulfan [?]7.2 mg/kg. Non-myeloablative regimens used any dose of ATG, fludarabine, cyclophosphamide, or TBI[?]200 cGy. (8) Fully HLA-matched unrelated BM or PB donors were matched at A, B, C, and DRB1 antigens by high resolution typing. For umbilical cord blood donors, full match was defined as $6 / 6$ HLA-antigen match (A, B, DRB1). A related donor was considered haploidentical if [?] 2 different antigens (A, B, C, or DRB1) were mismatched. CIBMTR gathers and reports acute GVHD grade following criteria published by Przepiorka et al. (9) Although currently CIBMTR gathers data on individual organ involvement with cGVHD based on NIH Consensus Criteria 2014; the data available through our platform contained only information on extent of cGVHD as limited or extensive based on definition by Shulman et al. (10) Limited cGVHD includes only localized skin involvement and/or liver dysfunction, while any other organ involvement is considered extensive.

\section{Statistical analyses}

Descriptive statistics were reported as median and range for continuous variables and frequencies and proportions for categorical variables. Comparisons for baseline patient characteristics between white and black groups were performed using permutation chi-square test or median test depending on the data type. The primary endpoint, overall survival (OS), was defined as the duration of time from transplant to time of death or the date of the last follow-up reported to the CIBMTR database. The overall survival was characterized using Kaplan-Meier plots and the log-rank test was used to examine differences in survival by race and HLA-match within each race type. A $P$ value of less than 0.05 was considered significant. All analyses were

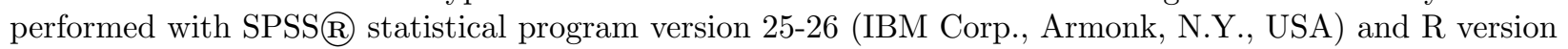
4.0 (R Core Team, 2020).

\section{Results}

A total of 316 children received transplant for HM during a 10-year period in one of the 5 FPBCC centers. Race was reported as White, Black, Asian and unknown in 238 (75.3\%), 57 (18\%), 9 (2.8\%) and $12(3.8 \%)$ patients, respectively. Subsequent analyses were done only for patients reported to the CIBMTR as W and $\mathrm{B}$, given very small numbers in other categories. Table 1 presents patient and transplant characteristics by race. There were no differences between $\mathrm{W}$ and $\mathrm{B}$ group in gender (male: $59 \% \mathrm{~W}, 60 \% \mathrm{~B}$ ); median age at transplant (9 years);,performance score ([?]90: $74 \% \mathrm{~W}, 79 \% \mathrm{~B})$; comorbidity number $(0-1: 78 \% \mathrm{~W}, 79 \% \mathrm{~B})$; disease status (CR1/CR2: $79 \% \mathrm{~W}, 75 \% \mathrm{~B}$ ); median time from diagnosis to transplant (14 months $\mathrm{W}, 11$ months B); use of bone marrow (BM), peripheral blood (PB), or umbilical cord blood (UCB) $(57 \%, 17 \%$, $26 \% \mathrm{~W}$ and $50 \%, 23 \%, 27 \% \mathrm{~B}$, respectively); donor-recipient gender match (matched: $51 \% \mathrm{~W}, 50 \% \mathrm{~B}$ ); donor-recipient CMV serology match (matched: $58 \% \mathrm{~W}, 63 \% \mathrm{~B}$ ); use of myeloablative regimens $(99 \% \mathrm{~W}$, $95 \% \mathrm{~B})$, and use of serotherapy $(47 \% \mathrm{~W}, 39 \% \mathrm{~B})$. There was a significant difference in the use of HLA-MMD $(53 \% \mathrm{~W}, 71 \% \mathrm{~B}, p=0.01)$. While the proportion of MMUD was identical $(16 \% \mathrm{~W}, 16 \% \mathrm{~B})$, there was an increased use MMRD and MMCBD among B $(13 \% \mathrm{~W}, 25 \% \mathrm{~B}$ and $19 \% \mathrm{~W}$ and $26 \% \mathrm{~B})$, respectively. When comparing HLA-MMD use to fully HLA-matched donors, B, compared to W, had RR of 1.47 [95\% CI 0.7-3] of receiving a mismatched unrelated donor $\mathrm{PB}$ or $\mathrm{BM}$, RR of 2.34 [95\% CI 1.2-4.4] of receiving HCT from a mismatched related donor, and a RR of 1.9 [95\% CI 0.99-3.6] of receiving a mismatched cord blood donor HCT.

As shown in Figure 1 Overall survival by race, there is no difference in survival between $\mathrm{W}$ and $\mathrm{B}$ children 
( $\log$-rank $p=0.72$ ). The 24-month overall survival was $61 \%$ [95\% CI 55-68\%] in W children and 60\% [95\% CI 48-75] in B children. Overall Survival by HLA-match indicated significantly better-2 year survival of W HLA-matched than HLA-mismatched transplant recipients $(72.2 \%$ [95\% CI 64.2-80.2] vs $51.6 \%$ [95\%CI 41.8-61.7], log-rank $p<0.01$ ) (Figure 1A). However, there was no difference in overall survival between HLAmatched and HLA-mismatched B HCT recipients (log-rank $p=0.94$ ) with 2-year survival of $60.2 \%$ [95\% CI 35-85] vs 59.3\% [95\% CI 43.2-75.6] (Figure 2B). Of note, only 16 B children received an HLA-matched HCT over 10-year period in Florida. Table 2 Transplant outcomes among White and Black Children shows that $\mathrm{W}$ and $\mathrm{B}$ recipients of MMRD HCT had higher 2-year survival rates than recipients of MMUD and MMCBD HCT; however, these differences were not statistically significant. Despite higher frequency of HLA-MMD among B children, there was no significant difference in the incidence of aGVHD (48\% W, 35\% $\mathrm{B}, p=0.1)$, incidence of cGVHD $(18.7 \% \mathrm{~W}, 28 \% \mathrm{~B}, p=0.1)$, and causes of death from recurrent disease and transplant-related toxicity $(16 \%, 22 \% \mathrm{~W}$ and $14 \%, 25 \% \mathrm{~B}, \mathrm{p}=0.9$, respectively), as outlined in Table 2 .

\section{Discussion}

Older studies, well summarized by Majhail et al indicate racial disparities associated with access to HCT as well as outcomes after HCT (11). Analyses of data from 1988-2002 including hospital discharge data, CIBMTR, SEER and US Census bureau data indicated that when compared to $\mathrm{W}$, adult B patients were less likely to receive HCT for hematologic malignancies. $(12,13)$ However, these differences in access to HCT were not present in younger patients. $(12,14)$ All patients in our study received HCT and thus had access to this tertiary center, high-cost care. We did not have data that would allow evaluation of access to transplant by race. Indirect evidence, such as racial distribution of our patients $(75 \% \mathrm{~W}, 18 \% \mathrm{~B}$ and $3 \%$ Asians), which correlated well with overall Florida population (2018 US Census Bureau estimates) comprising $75 \%$ W (53\% Non-Hispanic White), $16 \% \mathrm{~B}$ and $2.8 \%$ Asians, and time from diagnosis to transplant, which was actually 3 months shorter in B than $\mathrm{W}$ children, suggests that access to transplant was not an issue.

However, B and W HCT recipients do not have the same access to HLA-matched donors. Increased diversity in HLA haplotypes among B populations, and underrepresentation of minorities in national bone marrow registries contribute to difficulty in finding fully matched unrelated donors for B patients and other minority HCT-recipients. $(4,15)$ Only 16 B children $(29 \%)$ in our study received a fully HLA-matched HCT during this 10 -year period.

The largest study describing the effects of race and socioeconomic status on outcomes of unrelated donor HCT (1995-2004), which included 1600 children <20 years, did not differentiate outcomes based on age. (7) When adult and pediatric populations were analyzed together, Baker et al showed that African-Americans but not Asian or Hispanic HCT recipients of unrelated donor transplants had significantly worse overall survival than W. African-Americans as well as Hispanics had higher treatment-related mortality than W. Also, patients with median income in the lowest quartile across racial groups, had worse OS and higher risk of treatment related mortality (7). Our study indicated that the majority of B patients (71\%) and $53 \%$ of W patients received an HLA-mismatched/alternative donor HCT. This is similar to Baker's study where $78 \%$ of B and $54 \% \mathrm{~W}$ received an HCT from a MMUD (7). Despite a larger proportion of HLA-mismatched transplants among B children in our study, overall survival and other outcomes were not different between $\mathrm{W}$ and B HCT recipients. In addition, unlike $\mathrm{W}$, who had a significantly lower survival after HLA-mismatched HCT, which is well described in the literature, B HCT recipients had identical outcomes after HLA-matched and HLA-mismatched transplants. Only $16 \mathrm{~B}$ patients received a fully-HLA matched transplant over a 10 year period and survival rates in this group were likely skewed due to low patient numbers. Although more $\mathrm{B}$ than $\mathrm{W}$ patients received HLA-mismatched HCT, they were more likely to receive MMRD, than MMUD $\mathrm{BM}$ or PB. Black children receiving MMRD/haploidentical transplant had a 2-year survival rate of $70 \%$, that did not subsequently decline. Although we do not have details of GVHD prophylaxis for patients receiving alternative donor transplants, all 5 FPBCC centers utilize post-transplant cyclophosphamide (PTCy), while only one center has the access to in vitro T-cell depletion. Recent reports indicate improved outcomes of recipients of haplo-identical HCT using PTCY for GVHD prophylaxis. (16) Similar to our findings, a recent randomized study indicated improved overall survival in haploidentical HCT recipients with PTCY 
comparing with recipients of MMUCB transplant. (17) Although deaths due to relapse and treatment-related causes were not different between W and B HCT-recipients, we noticed an unacceptably high rate of death (22-25\%) due to treatment-related causes. Lowering those rates for all children will be one of the FPBCC priorities.

Despite limitations of our study including relatively small number of patient, lack of data on socio-economic status, ethnic origin, and lack of detailed data on GVHD prophylaxis, this is the largest contemporary pediatric study examining outcomes by race in children with HM undergoing HCT. Overall, our results are encouraging as they show that lack of HLA-matched donors did not translate into adverse HCT outcomes for B children.

\section{Conclusions}

In a contemporary cohort of children from Florida with HM, we found that B children had an increased likelihood of receiving an HLA-mismatched donor HCT, and in particular a mismatched related donor transplant. HLA-matching significantly affected 2-year survival among W but not among B HCT recipients. Despite increased use of mismatched donors, overall survival, incidence of GVHD and causes of death did not differ between $\mathrm{W}$ and $\mathrm{B}$ children receiving $\mathrm{HCT}$ for hematologic malignancies.

\section{Conflict of Interest Statement}

The authors of this manuscript have no competing interests to disclose.

\section{Acknowledgement}

We gratefully acknowledge the support of Dr. Douglas Rizzo, Senior Scientific Director from CIBMTR who introduced us to the eDBtC platform. We thank the nonprofit Children's Miracle Network for their 2018 and 2020 grants in support of FPBCC.

\section{.References}

1. Bhatia S, Sather H, Heerema N, Trigg M, Gaynon P and Robinson L. Racial and ethnic differences in survival of children with acute lymphoblastic leukemia. Blood. 2002;100(6):1957-1964.

2. Kadan-Lottick N, Ness K, Bhatia S, and Gurney J. Survival variability by race and ethnicity in childhood acute lymphoblastic leukemia. JAMA. 2003;290:2008-2014.

3. Goggins W, Lo FK. Racial and ethnic disparities in survival of US children with acute lymphoblastic leukemia: evidence from the SEER database 1988-2008. Cancer Causes Control.2012;23(5):737-43.

4. Kritane K, Lee S. Racial and ethnic disparities in hematologic malignancies. Blood 2017;130:1699-1705.

5. Aplenc R, Alonzo TA, Gerbingf RB, Smith FO, Meshinchi S, Ross JA, et al. Ethnicity and survival in childhood acute myeloid leukemia: a report from the Children's Oncology Group. Blood. 2006;108:7480 .

6. Bhatia S. Disparities in cancer outcomes: lessons learned from children with cancer. Pediatr Blood Cancer 2011.2011;56:994-1002.

7. Baker S, Davies S, Majhail N, Hassebroek A, Klein J, Ballen K, et al. Race and Socioeconomic status influence outcomes of unrelated donor hematopoietic cell transplantation. Biol Blood Marrow Transplant. 2009;15:1543-1554.

8. Bacigalupo A, Ballen K, Rizzo D, Giralt S, Lazarus H, Ho V, et al. Defining the intensity of conditioning regimens: Working Definitions. Biol Blood Marrow Transplant 2009; 15:1628-33.

9. Przepiorka D, Weisdorf D, Martin R, Klingemann HG, Beatty P, Hows J, et al. 1994 Consensus conference on Acute GVHD Grading. Bone Marrow Transplant.1995;15:825-8.

10. Shulman HM, Sullivan KM, Weiden PL, McDonald GB, Striker GE, Sale GE, et al. Chronic graftversus-host syndrome in man. A long-term clinicopathologic study of 20 Seattle patients. Am J Med.1980;69:204-17.

11. Majhail NS, Nayyar S, Santibanez B, Murphy EA, and Denzen EM. Racial disparities in hematopoietic cell transplantation in the United States. Bone Marrow Transplant 2012;47(11):doi.10.1038/bmt.2011.214. 
12. Joshua TV, Rizzo JD, Zhang MJ, Hari PN, Kurian S, Pasquini M, et al. Access to hematopoietic stem cell transplantation: effect of race and sex. Cancer. 2010; 116:3469-3476.

13. Mitchell JM, Meehan KR, Kong J, Schulman KA. Access to bone marrow transplantation for leukemia and lymphoma: the role of sociodemographic factors. J Clin Oncol. 1997;15:2644-2651.

14. Hwang JP, Lam TP, Cohen DS, Donato ML, Geraci JM. Hematopoietic stem cell transplantation among patients with leukemia of all ages in Texas. Cancer. 2004;101:2230-2238.

15. Oh H, Loberiza F, Zhang M-J, Ringden O, Akiyama H, Asai T, et al. Comparison of graft-versus-host disease and survival after HLA-identical sibling bone marrow transplantation in ethnic populations. Blood 2005;105:1408-1416.

16. Symons H, Zahurak M, Cao A, Chen A, Cooke K, Gamper C, et al. Myeloablative haploidentical BMT with posttransplant cyclophosphamide for hematologic malignancies in children and adults. Blood Adv 2020; 4:3913-3925.

17. Fuchs E, O'Donnell P, Eapen M, Logan B, Antin J, Dawson P, et al. Double unrelated umbilical cord blood vs HLA-haploidentical bone marrow transplantation: CTN 1101 trial. Blood 2021;137:420-428.

Figure Legends:Figure 1 Overall Survival by Race Figure 2 Overall Survival by HLA-Match, A - White HCT Recipients, B- Black HCT recipients

Data Access:

The data that support the findings of this study are available from the corresponding author upon a reasonable request.

\section{Hosted file}

TABLE 1 Patient and Transplant Characteristics by Race.pdf available at https://authorea. com/users/406895/articles/517376-race-as-a-factor-in-donor-selection-and-survival-ofchildren-with-hematologic-malignancies-undergoing-hematopoietic-stem-cell-transplant-inflorida

\section{Hosted file}

Table 2 Outcomes of Transplant.pdf available at https://authorea.com/users/406895/articles/ 517376-race-as-a-factor-in-donor-selection-and-survival-of-children-with-hematologicmalignancies-undergoing-hematopoietic-stem-cell-transplant-in-florida

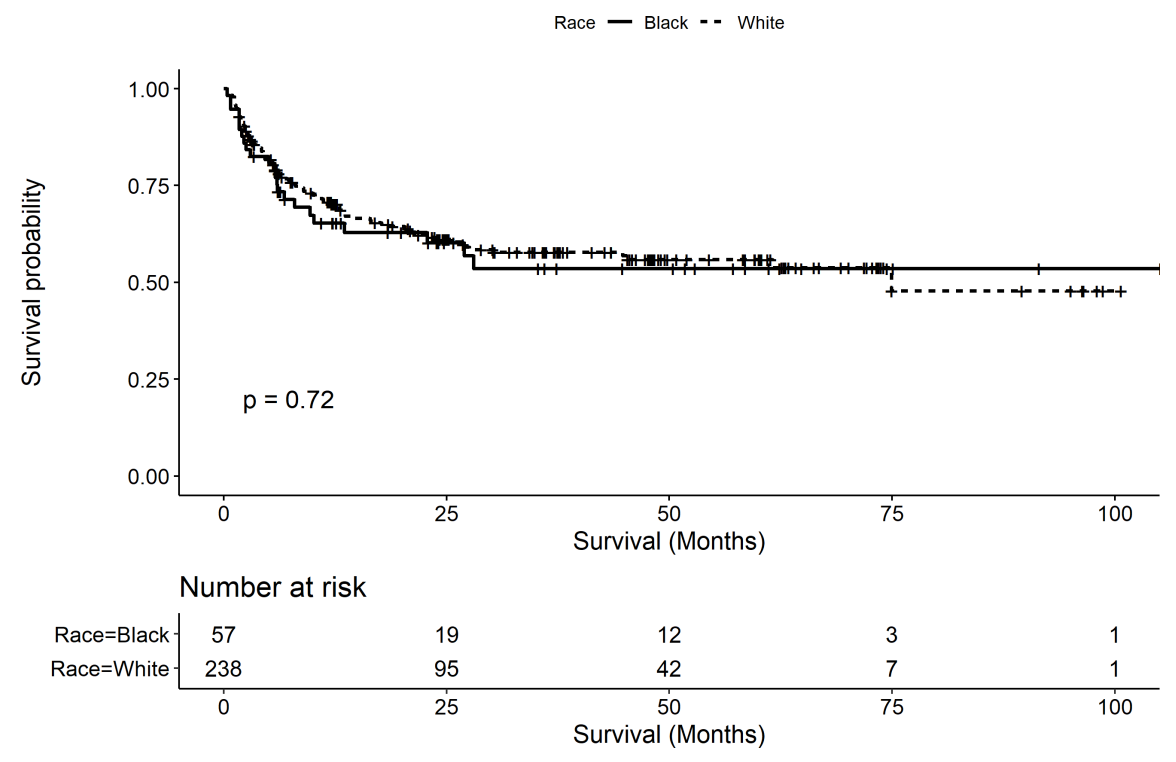


A

HLAMatch — HLA-matched - - mismatched

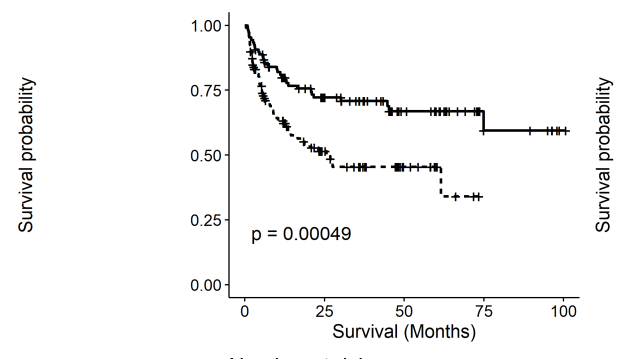

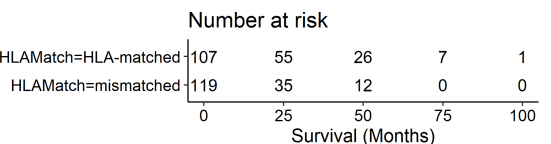

B

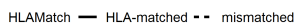

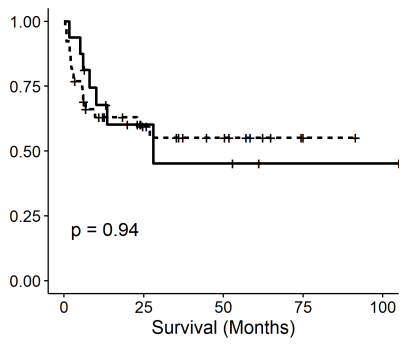

Number at risk

\begin{tabular}{|c|c|c|c|c|c|}
\hline \multicolumn{6}{|c|}{ Number at risk } \\
\hline HLAMatch=HLA-matched & 16 & 4 & 3 & 1 & 1 \\
\hline HLAMatch=mismatched & 39 & 15 & 9 & 2 & 0 \\
\hline & 0 & 25 & 50 & 75 & 100 \\
\hline
\end{tabular}

